\title{
Nicotinamide attenuates aquaporin 3 overexpression induced by retinoic acid through inhibition of EGFR/ERK in cultured human skin keratinocytes
}

\author{
XIUZU SONG ${ }^{1,2}$, AIE XU $^{1}$, WEI PAN ${ }^{2}$, BRITTANY WALLIN $^{2}$, REBECCA KIVLIN $^{2}$, \\ SHAN LU ${ }^{2}, \mathrm{CONGCAO}^{3}, \mathrm{ZHIGANG} \mathrm{BI}^{4}$ and YINSHENG WAN ${ }^{2}$ \\ ${ }^{1}$ Department of Dermatology, the Third Hospital of Hangzhou, Hangzhou 310009, P.R. China; \\ ${ }^{2}$ Department of Biology, Providence College, Providence, RI 02918; ${ }^{3}$ Department of Molecular \\ Microbiology and Immunology, Brown University, Providence, RI 02914, USA; ${ }^{4}$ Department of \\ Dermatology, the First Affiliated Hospital of Nanjing Medical University, Nanjing 210029, P.R. China
}

Received February 29, 2008; Accepted April 16, 2008

DOI: 10.3892/ijmm_00000013

\begin{abstract}
The most common adverse effects that are related to all-trans retinoic acid (atRA) treatment are irritation and dryness of the skin. atRA therapy is reported to impair barrier function as achieved by trans-epidermal water loss (TEWL). Treatment with nicotinamide prior to initiation of atRA therapy provides additional barrier protection and thus reduces susceptibility of retinoic acid. Our previous studies showed that atRA upregulates aquaporin 3 (AQP3) in cultured human skin keratinocytes and fibroblasts. Others have demonstrated that in atopic dermatitis, overexpression of AQP3 is linked to elevated TEWL and that nicotinamide treatment reduces skin TEWL. In this study, we observed that while atRA upregulates AQP3 expression in cultured human skin keratinocytes (HaCaT cells), nicotinamide attenuates the effect of atRA in a concentration-dependent manner. atRA treatment induces EGFR and ERK activation. PD153035, an EGFR inhibitor, and U0126, an ERK inhibitor, inhibit atRA-induced upregulation of AQP3. Nicotinamide also inhibits atRAinduced activation of EGFR/ERK signal transduction and decreases water permeability by downregulating AQP3 expression. Collectively, our results indicate that the effect of atRA on AQP3 expression is at least partly mediated by EGFR/ERK signaling in cultured human skin keratinocytes. Nicotinamide attenuates atRA-induced AQP3 expression through inhibition of EGFR/ERK signal transduction and
\end{abstract}

Correspondence to: Dr Aie Xu, Department of Dermatology, the Third Hospital of Hangzhou, 38 Xihu Street, Hangzhou 310009 , P.R. China

E-mail: xuaiehz@sina.com

Dr Yinsheng Wan, Department of Biology, Providence College, 549 River Avenue, Providence, RI 02918, USA

E-mail: yswan@providence.edu

Key words: aquaporin 3, retinoic acid, nicotinamide, EGFR, ERK eventually decreases water permeability and water loss. Our study provides insights into the molecular mechanism through which nicotinamide reverses the side effects of dryness in human skin after treatment with atRA.

\section{Introduction}

All-trans retinoic acid (atRA), a major biologically active retinoid, remains one of the most important regulators of skin function. Topical treatment with atRA improves the clinical and histological appearance of long-term photo-damaged skin (1). Although retinoic acid provides benefits to sunexposed skin, it is frequently accompanied by significant skin irritation which limits its clinical application. The most common side effects of retinoic acid treatment are irritation and dryness of the skin (2). The mechanisms leading to retinoid adverse effects have not been fully elucidated, but retinoic acid therapy has been demonstrated to impair barrier function as assessed by trans-epidermal water loss (TEWL) measurement (3-5).

Skin water transport has a close relationship with the expression of aquaporin 3 (AQP3) in skin keratinocytes. Recent studies have shown that AQP3-deficient mice show delayed skin barrier recovery, suggesting a possible involvement of AQP3 in the regulation of keratinocyte differentiation and proliferation (6). Moreover, inhibition of AQP3 activity significantly decreases epidermal water flux (7). In contrast, higher AQP3 expression is observed in young rat skin, which is thought to be associated with increased TEWL in young rats (8). Evidence also shows that higher expression of AQP3 is linked to increased TEWL in atopic eczema (AE), leading to skin dryness in AE (9). It has been demonstrated that atRA increases expression of AQP3 in normal human skin (10), suggesting that AQP3 is involved in the impairment of skin barrier function induced by atRA.

Clinically, some moisturizers have been proven to improve the skin barrier function of atopic dry skin. Nicotinamide, also known as vitamin B3, has been demonstrated to have beneficial effects on certain skin conditions, such as photo 
damage and cutaneous hyperpigmentation (11-14). Studies have shown that topical nicotinamide application on dry human skin results in increased levels of ceramide and free fatty acids in the epidermis, and decreased levels of TEWL, suggesting that nicotinamide improves the epidermal permeability barrier (15). Furthermore, myristyl nicotinate reduces TEWL and provides an additional barrier protection and tolerability of retinoic acid (16). However, the question whether nicotinamide retains skin barrier function by attenuating atRA-induced AQP3 overexpression remains to be answered.

The promotion of keratinocyte proliferation and differentiation by retinoids is partly mediated by the epidermal growth factor receptor (EGFR) signal pathway. EGFR activation plays a central role in retinoid-induced epidermal hyperplasia and EGFR inhibitors mitigate retinoid-induced scaling (17). The involvement of the EGFR pathway in atRA signaling was further demonstrated by increased phosphorylation of EGFR and its downstream signaling component ERK1/2 in our previous study (18). The evidence that ERK1/2 activation by atRA is abolished by EGFR inhibitor AG1478 strongly suggests that the EGFR and ERK1/2 signal pathway is involved in atRA actions in many cell types in vitro and in human skin epidermis in vivo (18-20). However, the question whether nicotinamide antagonizes atRA-induced AQP3 overexpression by inhibiting EGFR and ERK1/2 activation needs to be further addressed.

Thus, the aim of this study was to investigate whether nicotinamide treatment prior to atRA attenuates water permeability mediated by AQP3 in cultured human keratinocytes. Furthermore, we undertook this study to investigate whether the EGFR/ERK signal pathway is involved in AQP3 expression and whether nicotinamide decreases AQP3 expression by inhibiting EGFR/ERK activation.

\section{Materials and methods}

Cell culture. As described previously $(21,22)$, spontaneously immortalized human keratinocytes (HaCat cells) and human skin fibroblasts were maintained in a DMEM medium (Sigma, St. Louis, MO) supplemented with a $10 \%$ fetal bovine serum (Invitrogen, Carlsbad, CA), penicillin/streptomycin (1:100, Sigma) and $4 \mathrm{mM}$ L-glutamine (Sigma), in a $\mathrm{CO}_{2}$ incubator at $37^{\circ} \mathrm{C}$. For Western blotting, cells were reseeded in 6-well plates at a density of $1 \times 10^{5}$ cells $/ \mathrm{ml}$ with fresh complete culture medium. For determination of water permeability, cells were reseeded in an 8-well culture chamber at a density of $1 \times 10^{4}$ cells $/ \mathrm{ml}$ and were identified with the aid of a fluorescence microscope using the excitation wavelength of $480 \mathrm{~nm}$. Unless otherwise indicated, cultures were grown to 70-80\% confluence and then serum-starved overnight in DMEM medium prior to treatment.

Antibodies and inhibitors. Rabbit anti-aquaporin 3 (AQP3) was obtained from Chemicon (Temecula, CA), rabbit antiphospho-EGFR, rabbit anti-phospho-ERK and rabbit antiERK were obtained from Cell Signaling Technology (Danvers, MA). Rabbit anti-EGFR (1068), goat anti-rabbit IgG-HRP and goat anti-mouse IgG-HRP antibody were received from Santa Cruz Biotechnology (Santa Cruz, CA).
Monoclonal mouse anti- $\beta$-actin and all-trans retinoic acid (atRA) were obtained from Sigma. EGFR and ERK inhibitors, PD153035 and U0126, were obtained from CalbioChem (San Diego, CA).

Western blot analysis. As reported previously (22), cultured $\mathrm{HaCat}$ cells with and without treatment were washed with cold PBS and harvested by scraping into $150 \mu \mathrm{l}$ of RIPA buffer. Cell lysates were incubated in $4^{\circ} \mathrm{C}$ for $30 \mathrm{~min}$. Proteins $(20 \mu \mathrm{g})$ were denatured in 5X SDS-PAGE sample buffer for $5 \mathrm{~min}$ at $95^{\circ} \mathrm{C}$. The proteins were separated by 10 or $12 \%$ SDS-PAGE and transferred onto PVDF membrane (Millipore, Bedford, MA). Nonspecific binding was blocked with $10 \%$ dry milk in TBST for $1 \mathrm{~h}$ at room temperature. After blocking, membranes were incubated with specific antibodies in dilution buffer (2\% BSA in TBS) overnight at $4{ }^{\circ} \mathrm{C}$. The blots were incubated with horseradish peroxidaseconjugated anti-rabbit or anti-mouse $\operatorname{IgG}$ at appropriate dilutions and room temperature for $1 \mathrm{~h}$. Antibody binding was detected using an enhanced chemiluminescence (ECL) detection system (GE Biosciences, NJ) following the manufacturer's instructions, and visualized by autoradiography with hyperfilm.

RNA interference (RNAi) experiments. Custom SMARTpool ${ }^{\circledR}$ RNAi duplexes (more than four sequences) for AQP3 were chemically synthesized by Dharmacon (Lafayette, CO). As described previously (22), HaCaT cells were cultured in complete medium that did not contain antibiotics, for 4 days. Cells $\left(1 \times 10^{5}\right)$ were seeded into a 6 -well plate 1 day prior to transfection and cultured to $70-80 \%$ confluence the following day. For RNAi experiments, $12 \mu 1$ of FuGene6 (Roche Diagnostics, Indianapolis, IN) was diluted in $88 \mu 1$ of DMEM for $5 \mathrm{~min}$ in room temperature. Then $10 \mu \mathrm{l}$ of $20 \mu \mathrm{M}$ doublestranded RNAs for AQP3 RNAi was mixed with DMEM containing FuGene6 and incubated for $30 \mathrm{~min}$ at room temperature for complex formation. Finally the complex was added to the well containing $2 \mathrm{ml}$ medium with the final AQP3 siRNA concentration of $100 \mathrm{nM}$. AQP3 protein expression and water permeability was determined $24 \mathrm{~h}$ after treatment.

Measurement of water permeability. The water permeability was measured using a well established method as described previously (23-25). The coverslips with the HaCaT cells were mounted in a closed perfusion chamber on the stage of a Zeiss fluorescence microscope. HaCaT cells were loaded with calcein and the water permeability measurement was performed. Cells were loaded with calcein by a 5 -min exposure to $20 \mu \mathrm{M}$ calcein-acetoxymethyl ester (calcein-AM; Molecular Probes) in 300 mosM PBS (in mM: $137 \mathrm{NaCl}$, $0.9 \mathrm{CaCl}_{2}, 0.49 \mathrm{MgCl}_{2}, 2.7 \mathrm{KCl}, 1.5 \mathrm{KH}_{2} \mathrm{PO}_{4}, 8.1 \mathrm{Na}_{2} \mathrm{HPO}_{4}$, $\mathrm{pH}$ 7.35). The loading of the cells with calcein and incubation with the drugs was performed at $30^{\circ} \mathrm{C}$ for $10 \mathrm{~min}$. The subsequent water permeability measurements were carried out at $10^{\circ} \mathrm{C}$ to decrease water diffusion through the lipid bilayer of plasma membrane. To measure water permeability, the cells were perfused with cold 300 mosM PBS for 1 min, and then the perfusate was switched to 150 mosM PBS at the same temperature (in mM: $62 \mathrm{NaCl}, 0.9 \mathrm{CaCl}_{2}, 0.49 \mathrm{MgCl}_{2}$, 
A

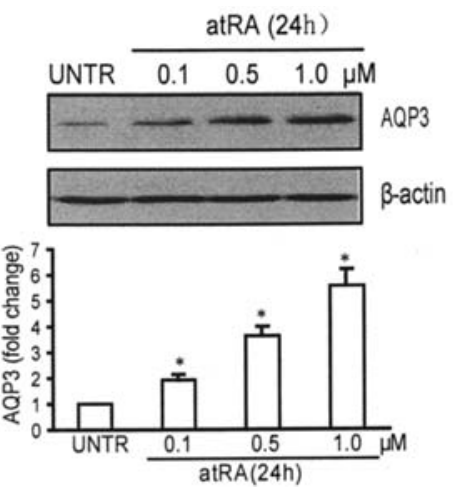

C
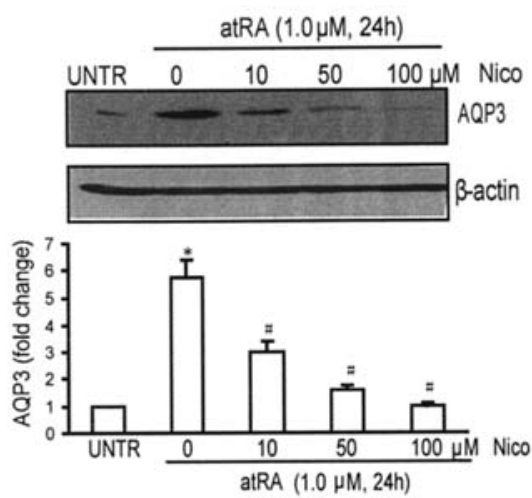

$\mathrm{E}$
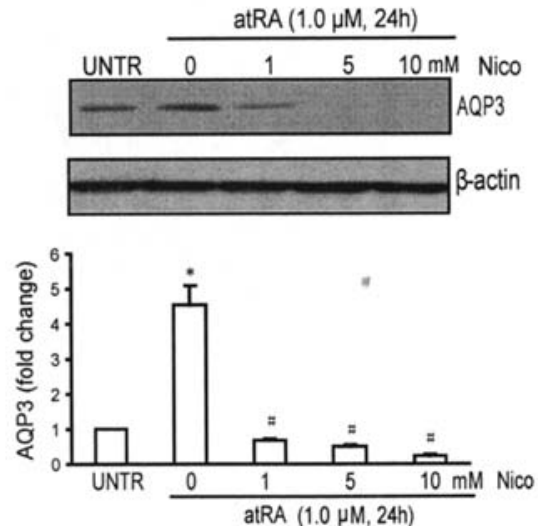

B
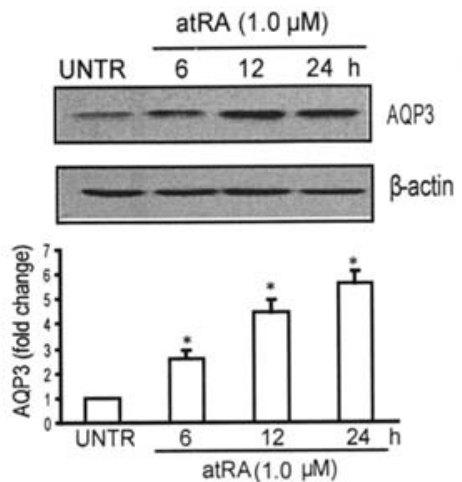

D
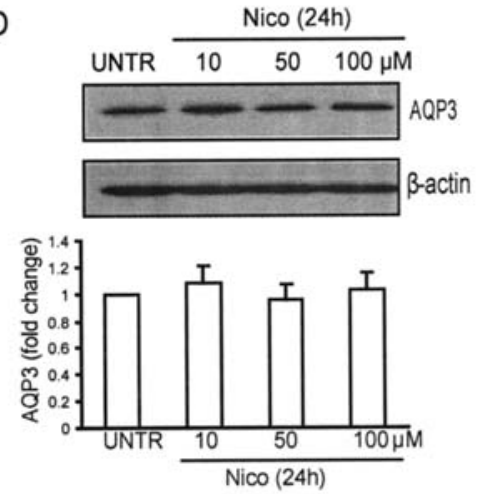

$\mathrm{F}$
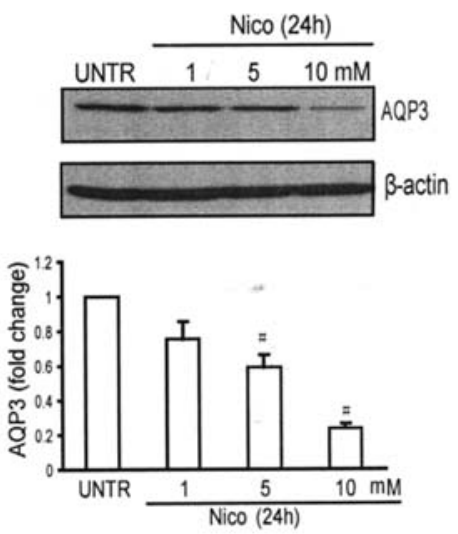

Figure1. Nicotinamide (Nico) inhibits all-trans retinoic acid (atRA)-induced upregulation of AQP3 in cultured human skin keratinocytes. HaCaT cells were treated with different concentrations of atRA $(0.1,0.5$ and $1.0 \mu \mathrm{M}$, shown in A) for $24 \mathrm{~h}$ or treated with atRA $(1.0 \mu \mathrm{M})$ for different time periods $(6,12$ and $24 \mathrm{~h}$, shown in B); AQP3 expression was measured by Western blotting and quantified and normalized to $B$-actin. HaCaT cells were treated with lower concentrations of Nico $(10,50$ and $100 \mu \mathrm{M}$, shown in D) alone or followed by atRA (1.0 $\mu \mathrm{M}$, shown in C) treatment for $24 \mathrm{~h}$; AQP3 was measured by Western blotting and quantified and normalized to $B$-actin. HaCaT cells were treated with higher concentrations of Nico $(1,5$ and $10 \mathrm{mM}$, shown in F) alone or followed by atRA $\left(1.0 \mu \mathrm{M}\right.$, shown in E) treatment for $24 \mathrm{~h}$; AQP3 was measured by Western blotting and quantified and normalized to $\beta$-actin. ${ }^{*} \mathrm{P}<0.05$ vs untreated (UNTR) group. ${ }^{~} \mathrm{P}<0.05$ vs $1.0 \mu \mathrm{M}$ atRA-treated group.

$\left.2.7 \mathrm{KCl}, 1.5 \mathrm{KH}_{2} \mathrm{PO}_{4}, 8.1 \mathrm{Na}_{2} \mathrm{HPO}_{4}, \mathrm{pH} 7.35\right)$. Fluorescence images were recorded every $30 \mathrm{sec}$ with excitation at $488 \mathrm{~nm}$ and emission at 515-525 $\mathrm{nm}$ (green) before and after the solution switch. The series of images was analyzed off-line by measuring the time course of the calcein fluorescence in cytoplasmic regions of individual cells. The half-life of deduction of fluorescence intensity was used as a comparable indicator of water permeability.

Statistical analysis. The values in the figures are expressed as the means \pm standard error (SE). The figures in this study are representative of 3 different experiments. Statistical analysis of the data between the control and treated groups was performed by a Student's t-test. Values of $\mathrm{p}<0.05$ were considered as statistically significant.

\section{Results}

Nicotinamide inhibits all-trans retinoic acid-induced upregulation of $A Q P 3$ in cultured keratinocytes. First, we examined whether atRA treatment induces AQP3 upregulation in cultured human skin keratinocytes. HaCaT cells were treated with different concentrations of atRA for $24 \mathrm{~h}$ and AQP 3 was measured by Western blot analysis. The results showed that 
A

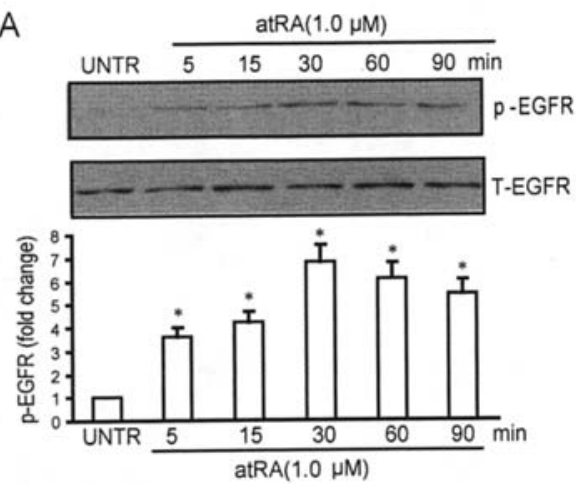

C
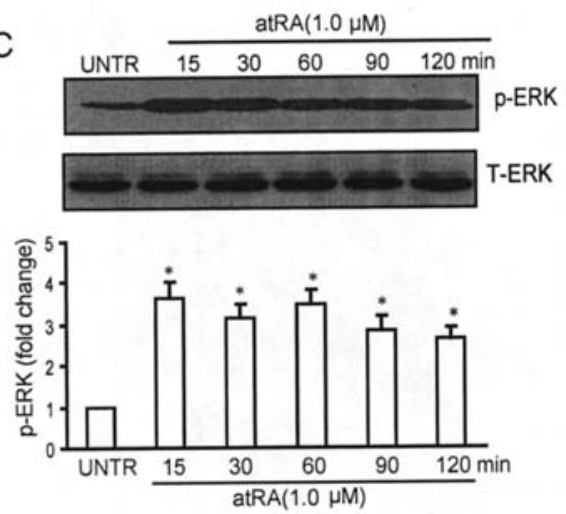

B
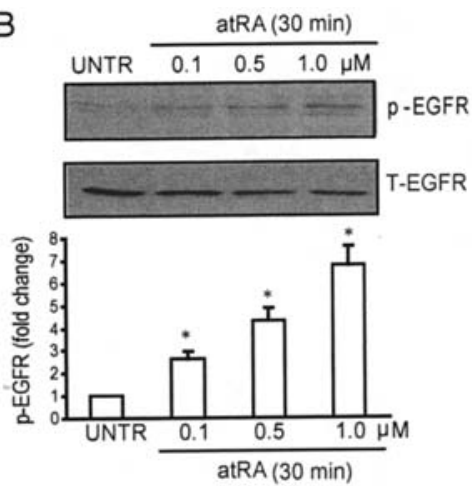

D
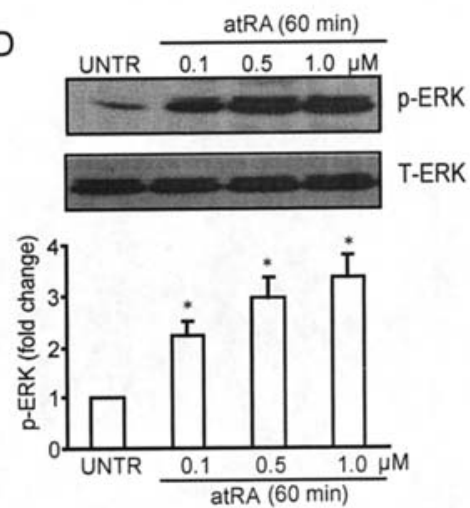

E
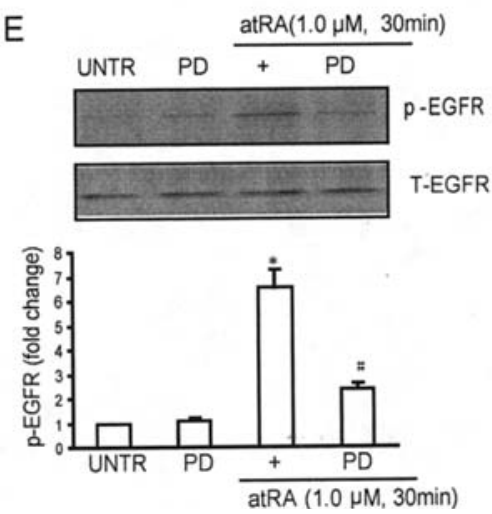

$\mathrm{F}$
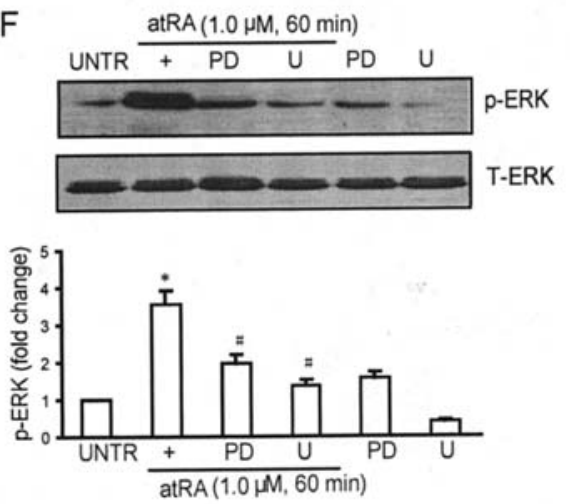

Figure 2. All-trans retinoic acid (atRA)-induced EGFR/ERK activation in cultured human skin keratinocytes (HaCaT cells). HaCaT cells were treated with atRA $(1 \mu \mathrm{M})$ for different time periods $(5,15,30,60$ and $90 \mathrm{~min}$, shown in A) or treated with different concentrations of atRA $(0.1,0.5$ and $1.0 \mu \mathrm{M}$, shown in B) for 30 min; phosphorylation EGFR (p-EGFR, Y1068) was analyzed by Western blotting and quantified and normalized to ß-actin. HaCaT cells were treated with atRA $(1.0 \mu \mathrm{M})$ for different time periods $(15,30,60,90$ and $120 \mathrm{~min}$, shown in $\mathrm{C})$ or treated with different concentrations of atRA (0.1, 0.5 and $1.0 \mu \mathrm{M}$, shown in D) for $60 \mathrm{~min}$; phosphorylation ERK (p-ERK) was analyzed by Western blotting and quantified and normalized to 3 -actin. HaCaT cells were pretreated with PD153035 (PD, $1.0 \mu \mathrm{M}$ ) or U0126 (U, $10 \mu \mathrm{M})$ for 1 h followed by atRA (1.0 $\mu \mathrm{M})$ treatment; phosphorylation EGFR (p-EGFR, Y1068) was analyzed by Western blotting $30 \mathrm{~min}$ after atRA treatment and quantified in E. Phosphorylation ERK (p-ERK) was analyzed by Western blotting 60 min after atRA treatment and quantified in $\mathrm{F} .{ }^{*} \mathrm{P}<0.05$ vs untreated (UNTR) group. ${ }^{\text {}} \mathrm{P}<0.05$ vs $1.0 \mu \mathrm{M}$ atRA-treated group.

atRA induces AQP3 upregulation in a concentration-dependent manner (Fig. 1A). A greater than five-fold increase in AQP3 expression was observed in $1.0 \mu \mathrm{M}$ atRA-treated cells, compared to untreated cells. atRA treatment also induced AQP3 upregulation in a time-dependent manner and the increased expression of AQP3 was observed as early as $6 \mathrm{~h}$ after atRA $(1.0 \mu \mathrm{M})$ treatment (Fig. 1B). We then investigated whether nicotinamide inhibited atRA-induced upregulation of AQP3. HaCaT cells were pretreated with different concentrations of nicotinamide followed by treatment with atRA $(1.0 \mu \mathrm{M})$ for $24 \mathrm{~h}$. Results showed that nicotinamide at all concentrations (10 $\mu \mathrm{M}-10 \mathrm{mM})$ inhibited AQP3 expression induced by atRA (Fig. 1C and E). Furthermore, nicotinamide treatment alone decreased AQP3 expression at high concentrations (1-10 mM, Fig. 1F), but the expression of AQP3 was not altered when cells were treated with nicotinamide alone at low concentrations (10 $\mu \mathrm{M}-100 \mu \mathrm{M})$ (Fig. 1D).

All-trans retinoic acid induces EGFR/ERK activation in cultured keratinocytes. Above data show that atRA treatment induced AQP3 upregulation in cultured human skin keratinocytes and our previous study showed that EGFR and ERK 
A
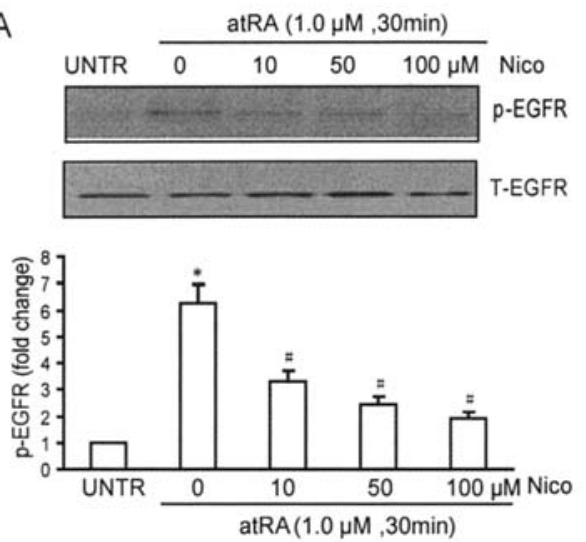

B
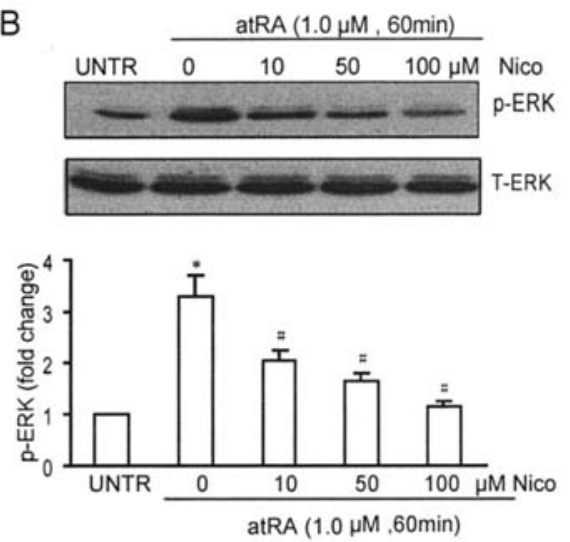

Figure 3. Nicotinamide (Nico) inhibits all-trans retinoic acid (atRA)-induced EGFR/ERK activation in cultured human skin keratinocytes. HaCaT cells were pretreated with different concentrations of Nico (10,50 and $100 \mu \mathrm{M})$ for $1 \mathrm{~h}$ followed by atRA (1.0 $\mu \mathrm{M})$ treatment; phosphorylation EGFR (p-EGFR, Y1068) was analyzed by Western blotting 30 min after atRA treatment and quantified in A. Phosphorylation ERK (p-ERK) was analyzed by Western blotting 60 min after atRA treatment and quantified in $\mathrm{B} .{ }^{*} \mathrm{P}<0.05$ vs untreated (UNTR) group. ${ }^{\text {}} \mathrm{P}<0.05$ vs $1.0 \mu \mathrm{M}$ atRA-treated group.

A
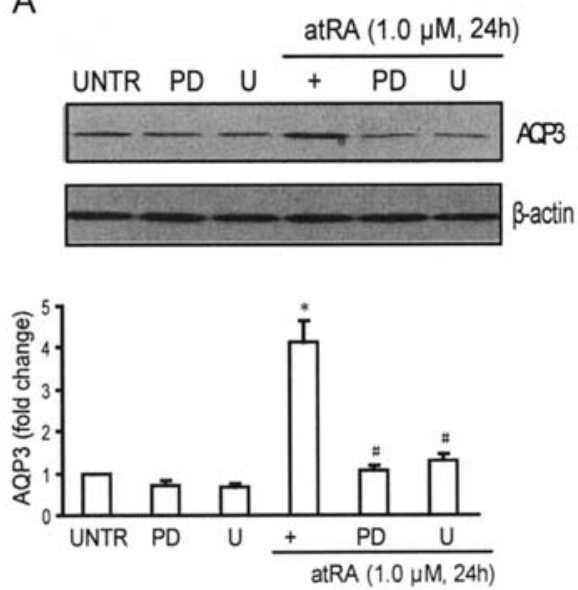
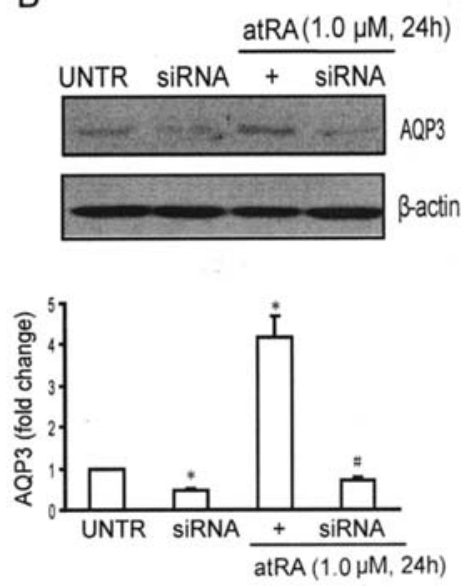

Figure 4. EGFR and ERK inhibitors block AQP3 upregulation induced by all-trans retinoic acid (atRA) in cultured human skin keratinocytes. HaCaT cells were pretreated with PD153035 (PD, $1.0 \mu \mathrm{M})$ or U0126 (U, $10 \mu \mathrm{M})$ for $1 \mathrm{~h}$ followed by atRA $(1.0 \mu \mathrm{M})$ treatment or not for $24 \mathrm{~h}$; AQP3 expression was analyzed by Western blotting and quantified in A. HaCaT cells were pretreated with siRNA (100 nM) followed by atRA ( $1.0 \mu \mathrm{M})$ treatment or not for $24 \mathrm{~h}$; AQP3 expression was analyzed by Western blotting and quantified in $\mathrm{B} .{ }^{*} \mathrm{P}<0.05$ vs untreated (UNTR) group. ${ }^{*} \mathrm{P}<0.05$ vs $1.0 \mu \mathrm{M}$ atRA-treated group.

were involved in AQP3 expression (22). In this study, we further investigated the signal transduction pathway of atRA-induced AQP3 expression. As shown in Fig. 2A and C, phosphorylation EGFR (p-EGFR, Y1068) and phosphorylation ERK (p-ERK) were induced by atRA $(1.0 \mu \mathrm{M})$ in a time-dependent manner. When HaCaT cells were treated with various concentrations of atRA $(0.1,0.5$, and $1.0 \mu \mathrm{M})$, the activation levels of p-EGFR and p-ERK were in an atRA concentration-dependent manner (Fig. 2B and D). To further verify the involvement of the EGFR/ERK pathway, cells were pretreated with EGFR inhibitor PD153035 and ERK inhibitor U0126 for $1 \mathrm{~h}$, followed by atRA $(1.0 \mu \mathrm{M})$ treatment. The results showed that p-EGFR activation was inhibited by PD153035 (Fig. 2E) and p-ERK activation was inhibited by both PD153035 and U0126 (Fig. 2F). These results support the notion that EGFR/ERK the pathway is involved in signal transduction in $\mathrm{HaCaT}$ cells treated with atRA.

Nicotinamide inhibits all-trans retinoic acid-induced EGFR/ERK activation in cultured keratinocytes. Above data show that nicotinamide inhibits atRA-induced upregulation of AQP3 at various concentrations and EGFR/ERK signal transduction pathway is involved in atRA-treated HaCaT cells. However, the question whether nicotinamide inhibits EGFR/ERK activation induced by atRA remains unanswered. To further examine the inhibitory role of nicotinamide in EGFR/ERK activation, HaCaT cells were pretreated with different concentrations of nicotinamide for $1 \mathrm{~h}$ followed by atRA $(1.0 \mu \mathrm{M})$ treatment. Results from Western blot analysis showed that EGFR and ERK activation was inhibited by nicotinamide at various concentrations $(10,50$, and $100 \mu \mathrm{M})$ with less than $30 \%$ of p-EGFR and $50 \%$ of p-ERK observed in cells pretreated with nicotinamide $(100 \mu \mathrm{M})$ compared with cells treated with atRA $(1.0 \mu \mathrm{M})$ alone (Fig. 3A and B).

EGFR and ERK inhibitors block AQP3 upregulation induced by all-trans retinoic acid in cultured keratinocytes. Our data above demonstrated that nicotinamide inhibits EGFR/ERK activation and AQP3 expression induced by atRA treatment in $\mathrm{HaCaT}$ cells. However, whether EGFR/ERK activation is 
A

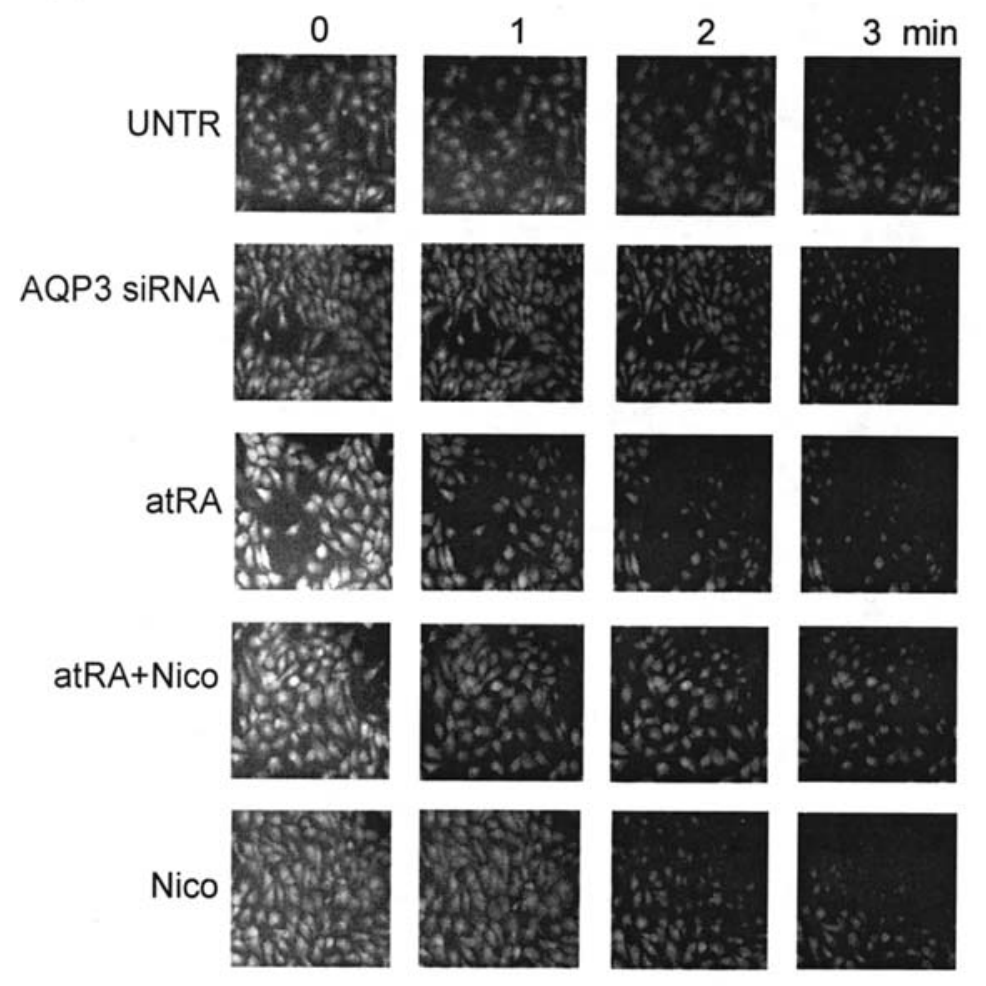

B

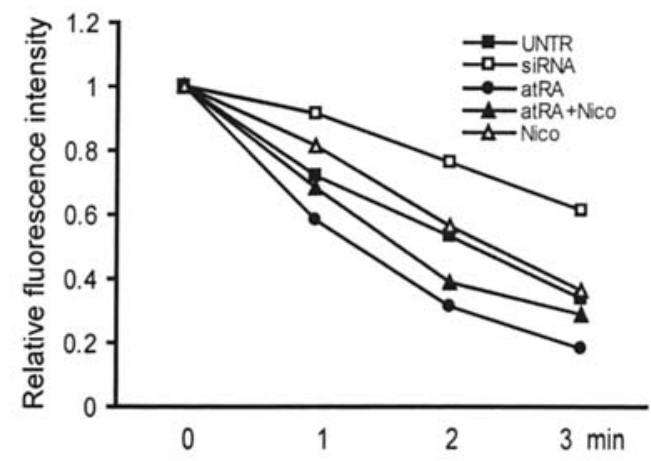

C

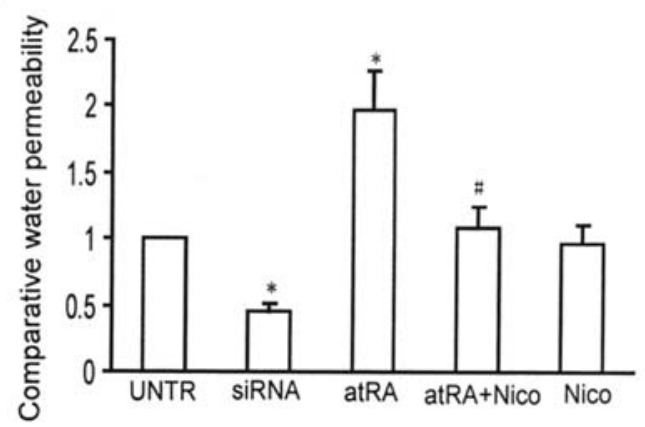

Figure 5. Nicotinamide (Nico) inhibits all-trans retinoic acid (atRA) induced water permeability mediated by AQP3 in cultured human skin keratinocytes. $\mathrm{HaCaT}$ cells were treated with atRA $(1.0 \mu \mathrm{M})$ for $24 \mathrm{~h}$ with or without Nico $(100 \mu \mathrm{M})$ pretreatment for $1 \mathrm{~h}$. Cell water permeability was analyzed by the method described in Materials and methods (shown in A). The water permeability of AQP3 siRNA transfected HaCaT cells is also shown in A. The relative fluorescence intensity is quantified in B. The half-life of deduction of fluorescence intensity is used here as a comparable indicator of water permeability (C). For the cell water permeability experiment at least 50 cells in 6 random views were quantified for relative fluorescence intensity. ${ }^{*} \mathrm{P}<0.05$ vs untreated (UNTR) group. ${ }^{\#} \mathrm{P}<0.05$ vs $1.0 \mu \mathrm{M}$ atRA-treated group. Magnification x200.

involved in the regulation of AQP3 expression has not been studied. To investigate this, HaCaT cells were pretreated with PD153035 and U0126 for $1 \mathrm{~h}$ followed by atRA $(1.0 \mu \mathrm{M})$ treatment for $24 \mathrm{~h}$, and AQP3 expression was analyzed by Western blotting. Results showed that PD153035 and U0126, which block activation of EGFR and ERK, inhibit AQP3 expression induced by atRA (Fig. 4A). These data support the notion that the EGFR/ERK pathway is involved in upregulation of AQP3 by atRA. As expected, AQP3 siRNA attenuates AQP3 expression induced by atRA $(1.0 \mu \mathrm{M})$ treatment (Fig. 4B).

Nicotinamide inhibits all-trans retinoic acid induced water permeability mediated by AQP3 in cultured keratinocytes. Previous studies have shown that AQP3 is involved in water permeability. We tested whether atRA-induced AQP3 upregulation leads to increased water permeability in cultured keratinocytes and whether nicotinamide has inhibitory effect. HaCaT cells were treated with atRA $(1.0 \mu \mathrm{M})$ for $24 \mathrm{~h}$ with or without nicotinamide $(100 \mu \mathrm{M})$ pretreatment for $1 \mathrm{~h}$. Cell water permeability was analyzed by the method described above. Results showed that atRA treatment increased water permeability and nicotinamide pretreatment decreased water permeability induced by atRA. Moreover, the water permeability of $\mathrm{HaCaT}$ cells transfected with AQP3 siRNA is decreased significantly, which supports the notion that AQP3 is involved in the regulation of cell water permeability (Fig. 5A-C).

\section{Discussion}

atRA and its derivatives, commonly called retinoids, are widely used in cosmetics for the prevention of skin aging. atRA is capable of regulating keratinocyte proliferation and differentiation and increasing stratum granulosum thickness $(26,27)$. However, retinoid treatment leads to skin dryness, irritation and peeling, which are caused by the impairment of skin barrier function $(28,29)$. These side effects limit the application of retinoid therapy. It would therefore be beneficial to develop certain moisturizers to attenuate the adverse effects of retinoids and preserve the skin barrier function. Nicotinomide, an essential dietary vitamin whose deficiency leads to pellagra, is commonly used to slow skin aging $(30,31)$. However, whether nicotinamide attenuates atRAinduced skin dryness and prevents water loss in the epidermis remains to be determined. Also, little is known about the underlying molecular mechanism of nicotinamide in skin physiology. Our study demonstrated that nicotinamide attenuates atRA-induced overexpression of AQP3 in cultured human skin keratinocytes and decreases water permeability. We also provided evidence that nicotinamide inhibits atRAinduced EGFR and ERK activation. This novel finding 
provides an insight into the molecular mechanism of nicotinamide's beneficial effect in preventing water loss due to the application of atRA.

AQP3 is the predominant aquaporin in the skin. Moreover, the expression of AQP3 is far higher in skin than in other tissues, indicating a vital role of AQP3 in skin water and glycerol transport $(7,32)$. Recent studies have indicated that atRA induces AQP3 overexpression at both gene and protein levels when applied topically on human skin, and elevated AQP3 expression leads to a burst of glycerol uptake $(10,32)$. Our previous study also showed that atRA attenuates UV radiation-induced AQP3 downregulation in cultured human skin keratinocytes, and that upregulation of AQP3 by atRA is accompanied by an increase of water transport (18). We confirmed in this study that atRA treatment increases the expression of AQP3 in a concentration-dependent manner, which leads to increased water permeability. The data that AQP3 siRNA treatment reduces water permeability support the notion that AQP3 plays an important role in cellular water transport in keratinocytes.

There are contradictory viewpoints about whether overexpression of AQP3, at least in part, accounts for skin dryness. Phenotypical studies in AQP3 null mice indicate that AQP3 plays an important role in epidermal glycerol and water transport, and upregulation of AQP3 leads to more water movement from dermis to epidermis $(6,7)$. In AQP3-deficient mice, skin barrier recovery and wound healing is significantly delayed, while upregulation of AQP3 facilitates epidermal cell migration during wound healing $(33,34)$. However, hyperexpression of AQP3 is also associated with the increase of TEWL. If the water-holding capacity of the stratum corneum is decreased, the increased water transport to the stratum corneum may lead to more water loss and skin dryness. This viewpoint is supported by a study in atopic eczema which is characterized by dry skin (35-37). Also, increased AQP3 expression is found in the epidermis of patients with atopic eczema (AE) in contrast with that of healthy skin. Patients with AE show defective skin barrier function and reduced water-holding capacity in stratum corneum which may contribute to increased water loss and dry skin in AE (9). In addition, published data also show that AQP3 expression influences skin hydration and contributes to increased water loss through rat immature skin (8). These findings demonstrate that increased expression of AQP3 may lead to increased water loss in AE. Our data show that atRA upregulates the expression of AQP3. This may further explain why atRA therapy induces skin dryness when it is used topically.

In this study, we found that nicotinamide decreased the expression of AQP3 and water permeability induced by atRA in a concentration-dependent manner. Our findings suggest that nicotinamide may be used as a moisturizer by downregulating the expression of AQP3 in keratinocytes. Previous studies have shown that topical nicotinamide improves skin barrier function, and nicotinamide cream is an effective moisturizer on atopic dry skin and may be used as an auxiliary medicine to treat atopic dermatitis $(38,39)$. Administration with myristyl nicotinate for one month reduces skin TEWL and provides additional barrier protection and tolerability of retinoic acid without interfering with improving efficacy (16).
In addition, multiple clinical studies have shown that topical nicotinamide treatment is well tolerated by skin and the appearance of aging facial skin, such as wrinkles, yellowing and elasticity, are all significantly improved $(30,40)$. Moreover, nicotinamide treatment increases the levels of ceramide and free fatty acids in the epidermis, suggesting that nicotinamide improves the epidermal permeability barrier (15). Collectively, this suggests that nicotinamide attenuates the dryness of skin induced by atRA by inhibiting the expression of AQP3.

The regulation of AQP3 expression by nicotinamide treatment remains unknown. Our data showed that atRA upregulates AQP3 expression through the activation of EGFR and ERK. Nicotinamide treatment inhibits the activation of EGFR/ERK and the expression of AQP3. In order to confirm that the EGFR/ERK signal pathway is involved in regulating AQP3 expression, we pretreated keratinocytes with EGFR and ERK inhibitors, PD153035 and U0126, followed by atRA treatment. Results showed that AQP3 was downregulated by PD153035 and U0126, which blocks the activation of EGFR and ERK. The involvement of the EGFR pathway in atRA signaling has been also demonstrated by the increased phosphorylation of EGFR and its downstream signaling component ERK1/2 in previous studies (17-19). Combined with our present study, we conclude that nicotinamide decreases water permeability mediated by AQP3. The downregulation of AQP3 by nicotinamide is through the inhibition of EGFR/ERK signal transduction which is activated by atRA. However, our data only showed that nicotinamide reduced atRA-induced high water permeability in cultured human skin keratinocytes in vitro. They do not fully elucidate the protective mechanism of nicotinamide when it is used topically on human skin. Further study will be carried out focusing on the protective effects of nicotinamide in vivo and in $\mathrm{AE}$ patients.

In conclusion, nicotinamide decreased the expression of AQP3 induced by atRA in a concentration-dependent manner in cultured human skin keratinocytes. atRA upregulated AQP3 expression through the activation of EGFR and ERK which was inhibited by nicotinamide. The water permeability decrease was accompanied by downregulation of AQP3. Our results demonstrate that nicotinamide attenuates atRA-induced AQP3 hyperexpression by inhibiting EGFR/ERK signal transduction with a result of decrease in water permeability. This study provides insight into the molecular mechanism through which nicotinamide attenuates the side effects of skin dryness due to topical application of atRA. As atRA and nicotinamide are both used in cosmetic products, the protective effects of nicotinamide against the side effects of atRA may enhance the anti-aging efficacy of atRA on skin.

\section{Acknowledgements}

This research was supported in part by a grant from NIH (P20 RR016457 from INBRE Program of the National Center for Research Resources to YSW), a grant from the National Natural Science Foundation of China (no. 30671896 to AX), a grant from the Board of Health, Hangzhou City (no. $2005 Z 0005$ to XZS), and a grant of the National Natural Science Foundation of China (no. 30671849 to ZGB). 


\section{References}

1. Fisher GJ and Voorhees JJ: Molecular mechanisms of photoaging and its prevention by retinoic acid: ultraviolet irradiation induces MAP kinase signal transduction cascades that induce Ap-1-regulated matrix metalloproteinases that degrade human skin in vivo. J Investig Dermatol Symp Proc 3: 61-68, 1998.

2. Lowe N, Gifford M, Tanghetti E, Poulin Y, Goldman M, Tse Y, Yamauchi P, Rosenzweig $\mathrm{H}$ and Kang S: Tazarotene 0.1\% cream versus tretinoin $0.05 \%$ emollient cream in the treatment of photodamaged facial skin: a multicenter, double-blind, randomized, parallel-group study. J Cosmet Laser Ther 6: 79-85, 2004.

3. Effendy I, Weltfriend S, Patil S and Maibach HI: Differential irritant skin responses to topical retinoic acid and sodium lauryl sulphate: alone and in crossover design. Br J Dermatol 134: 424-430, 1996.

4. Tagami H, Tadaki T, Obata M and Koyama J: Functional assessment of the stratum corneum under the influence of oral aromatic retinoid (etretinate) in guinea-pigs and humans. Comparison with topical retinoic acid treatment. Br J Dermatol 127: 470-475, 1992.

5. Ale SI, Laugier JP and Maibach HI: Differential irritant skin responses to tandem application of topical retinoic acid and sodium lauryl sulphate: II. Effect of time between first and second exposure. Br J Dermatol 137: 226-233, 1997.

6. Hara M, Ma T and Verkman AS: Selectively reduced glycerol in skin of aquaporin-3-deficient mice may account for impaired skin hydration, elasticity, and barrier recovery. J Biol Chem 277: 46616-46621, 2002.

7. Sougrat R, Morand M, Gondran C, Barre P, Gobin R, Bonte F, Dumas $\mathrm{M}$ and Verbavatz JM: Functional expression of AQP3 in human skin epidermis and reconstructed epidermis. J Invest Dermatol 118: 678-685, 2002

8. Agren J, Zelenin S, Hakansson M, Eklof AC, Aperia A, Nejsum LN, Nielsen S and Sedin G: Transepidermal water loss in developing rats: role of aquaporins in the immature skin. Pediatr Res 53: 558-565, 2003.

9. Olsson M, Broberg A, Jernas M, Carlsson L, Rudemo M, Suurkula M, Svensson PA and Benson M: Increased expression of aquaporin 3 in atopic eczema. Allergy 61: 1132-1137, 2006.

10. Bellemere G, Von Stetten O and Oddos T: Retinoic acid increases aquaporin 3 expression in normal human skin. J Invest Dermatol 128: 542-548, 2008.

11. Jacobson EL, Kim H, Kim M, Williams JD, Coyle DL, Coyle WR, Grove G, Rizer RL, Stratton MS and Jacobson MK: A topical lipophilic niacin derivative increases NAD, epidermal differentiation and barrier function in photodamaged skin. Exp Dermatol 16: 490-499, 2007

12. Tashtoush BM, Kim H, Jacobson EL and Jacobson MK: Formulation compatibility of myristyl nicotinate with drugs used to treat dermatological conditions involving an impaired skin barrier. Drug Dev Ind Pharm 33: 1176-1182, 2007.

13. Greatens A, Hakozaki T, Koshoffer A, Epstein H, Schwemberger S, Babcock G, Bissett D, Takiwaki H, Arase S, Wickett RR and Boissy RE: Effective inhibition of melanosome transfer to keratinocytes by lectins and niacinamide is reversible. Exp Dermatol 14: 498-508, 2005.

14. Gehring W: Nicotinic acid/niacinamide and the skin. J Cosmet Dermatol 3: 88-93, 2004.

15. Tanno O, Ota Y, Kitamura N, Katsube $\mathrm{T}$ and Inoue $\mathrm{S}$ : Nicotinamide increases biosynthesis of ceramides as well as other stratum corneum lipids to improve the epidermal permeability barrier. Br J Dermatol 143: 524-531, 2000.

16. Jacobson MK, Kim H, Coyle WR, Kim M, Coyle DL, Rizer RL and Jacobson EL: Effect of myristyl nicotinate on retinoic acid therapy for facial photodamage. Exp Dermatol 16: 927-935, 2007.

17. Rittie L, Varani J, Kang S, Voorhees JJ and Fisher GJ: Retinoid-induced epidermal hyperplasia is mediated by epidermal growth factor receptor activation via specific induction of its ligands heparin-binding EGF and amphiregulin in human skin in vivo. J Invest Dermatol 126: 732-739, 2006.

18. Cao C, Wan S, Jiang Q, Amaral A, Lu S, Hu G, Bi Z, Kouttab N, Chu W and Wan Y: All-trans retinoic acid attenuates ultraviolet radiation-induced down-regulation of aquaporin-3 and water permeability in human keratinocytes. J Cell Physiol, on line, 2007.
19. Pasonen-Seppanen SM, Maytin EV, Torronen KJ, Hyttinen JM, Hascall VC, Maccallum DK, Kultti AH, Jokela TA, Tammi MI and Tammi RH: All-trans retinoic acid-induced hyaluronan production and hyperplasia are partly mediated by EGFR signaling in epidermal keratinocytes. J Invest Dermatol 128: 797-807, 2008.

20. Canon E, Cosgaya JM, Scsucova S and Aranda A: Rapid effects of retinoic acid on CREB and ERK phosphorylation in neuronal cells. Mol Biol Cell 15: 5583-5592, 2004.

21. Zhang QS, Maddock DA, Chen JP, Heo S, Chiu C, Lai D, Souza K, Mehta S and Wan YS: Cytokine-induced p38 activation feedback regulates the prolonged activation of AKT cell survival pathway initiated by reactive oxygen species in response to UV irradiation in human keratinocytes. Int J Oncol 19: 1057-1061, 2001.

22. Cao C, Sun Y, Healey S, Bi Z, Hu G, Wan S, Kouttab N, Chu W and Wan Y: EGFR-mediated expression of aquaporin-3 is involved in human skin fibroblast migration. Biochem J 400: 225-234, 2006.

23. Zelenina M, Bondar AA, Zelenin S and Aperia A: Nickel and extracellular acidification inhibit the water permeability of human aquaporin-3 in lung epithelial cells. J Biol Chem 278: 30037-30043, 2003.

24. Zelenina M, Tritto S, Bondar AA, Zelenin S and Aperia A: Copper inhibits the water and glycerol permeability of aquaporin-3. J Biol Chem 279: 51939-51943, 2004

25. Zelenina M, Zelenin S, Bondar AA, Brismar $\mathrm{H}$ and Aperia A: Water permeability of aquaporin- 4 is decreased by protein kinase $\mathrm{C}$ and dopamine. Am J Physiol Renal Physiol 283: F309-F318, 2002.

26. Tur E, Hohl D, Jetten A, Panizzon R and Frenk E: Modification of late epidermal differentiation in photoaged skin treated with topical retinoic acid cream. Dermatology 191: 124-128, 1995.

27. Kimura T and Doi K: Effects of all-trans-retinoic acid on the dorsal skin of hairless dogs. Toxicol Pathol 26: 595-601, 1998.

28. Weiss JS, Shavin JS, Nighland M and Grossman R: Tretinoin microsphere gel $0.1 \%$ for photodamaged facial skin: a placebocontrolled trial. Cutis 78: 426-432, 2006.

29. Phillips TJ: An update on the safety and efficacy of topical retinoids. Cutis 75: 14-22, 2005 .

30. Chiu PC, Chan CC, Lin HM and Chiu HC: The clinical antiaging effects of topical kinetin and niacinamide in Asians: a randomized, double-blind, placebo-controlled, split-face comparative trial. J Cosmet Dermatol 6: 243-249, 2007.

31. Kobayashi $\mathrm{M}$ and Shimizu S: Nicotinic acid and nicotinamide. Nippon Rinsho 57: 2211-2217, 1999.

32. Hara-Chikuma $M$ and Verkman AS: Aquaporin-3 functions as a glycerol transporter in mammalian skin. Biol Cell 97: 479-486, 2005 .

33. Hara-Chikuma M and Verkman AS: Aquaporin-3 facilitates epidermal cell migration and proliferation during wound healing. J Mol Med 86: 221-231, 2008.

34. Levin MH and Verkman AS: Aquaporin-3-dependent cell migration and proliferation during corneal re-epithelialization. Invest Ophthalmol Vis Sci 47: 4365-4372, 2006.

35. Eberlein-Konig B, Schafer T, Huss-Marp J, Darsow U, Mohrenschlager M, Herbert O, Abeck D, Kramer U, Behrendt H and Ring J: Skin surface $\mathrm{pH}$, stratum corneum hydration, transepidermal water loss and skin roughness related to atopic eczema and skin dryness in a population of primary school children. Acta Derm Venereol 80: 188-191, 2000.

36. Hon KL, Wong KY, Leung TF, Chow CM and Ng PC: Comparison of skin hydration evaluation sites and correlations among skin hydration, transepidermal water loss, SCORAD index, Nottingham Eczema Severity Score, and quality of life in patients with atopic dermatitis. Am J Clin Dermatol 9: 45-50, 2008.

37. Seidenari S and Giusti G: Objective assessment of the skin of children affected by atopic dermatitis: a study of $\mathrm{pH}$, capacitance and TEWL in eczematous and clinically uninvolved skin. Acta Derm Venereol 75: 429-433, 1995.

38. Soma Y, Kashima M, Imaizumi A, Takahama H, Kawakami T and Mizoguchi M: Moisturizing effects of topical nicotinamide on atopic dry skin. Int J Dermatol 44: 197-202, 2005.

39. Bissett D: Topical niacinamide and barrier enhancement. Cutis 70: 8-12, 2002.

40. Bissett DL, Oblong JE and Berge CA: Niacinamide: A B vitamin that improves aging facial skin appearance. Dermatol Surg 31: 860-865, 2005. 